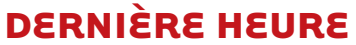

\section{La reprogrammation directe}

\section{Les transitions cellulaires dévoilées par un petit ver}

Marie-Charlotte Morin, Sophie Jarriault
IGBMC, CNRS UMR7104, Inserm U964, Université de Strasbourg, 1, rue Laurent Fries, BP 10142, 67404 IIIkirch CU Strasbourg, France. sophie@igbmc.fr
La transdifférenciation: une alternative prometteuse pour la médecine régénérative Améliorer ou pallier les faibles capacités de régénération de nos tissus et organes après une amputation, ou lors d'une maladie dégénérative, est un enjeu majeur de santé publique. Les solutions actuelles, comme la greffe d'organes, présentent des inconvénients considérables: faible disponibilité des greffons, nécessaire compatibilité immunologique. Malgré la formidable capacité des cellules souches embryonnaires (ES) à générer tous les types cellulaires, les problèmes éthiques liés à leur origine font de leur manipulation une technique très controversée. Ainsi, la possibilité de transformer une cellule déjà différenciée en un autre type cellulaire ouvre de nombreux champs d'applications thérapeutiques prometteurs. Les cellules saines du patient serviraient à la régénération d'autres cellules ou tissus endommagés tout en éliminant les problèmes de compatibilité et éthiques. Pendant longtemps, une hypothèse ayant valeur de dogme attribuait un caractère figé à l'identité finale d'une cellule, ce qui a nourri un certain scepticisme à l'égard de la possible plasticité de cellules déjà différenciées $[16,17](\rightarrow)$. Une théorie scientifique en vogue jusqu'aux années 1950, selon laquelle le matériel génétique des cellules serait altéré irréversiblement au cours de la différenciation cellulaire, a néanmoins connu un revers dès 1958 avec les expériences de transfert de noyau somatique [1]. La cellule n'est donc pas condamnée à un destin figé. En conséquence, la communauté scientifique a développé un intérêt exponentiel pour les stratégies permettant la manipulation du potentiel des cellules et la reprogrammation de l'identité cellulaire par conversion d'une cellule déjà différenciée en un autre type cellulaire.

Ces dernières années, la reprogrammation cellulaire a connu un essor retentissant grâce à la découverte que quatre facteurs seulement permettent la dédifférenciation d'une cellule différenciée en cellule souche pluripotente induite (iPS) $[2,14]$. Ces cellules iPS peuvent ensuite être dirigées vers un autre destin cellulaire. Cependant, le grand nombre d'études dédiées à ces cellules a permis d'identifier les risques potentiels d'utilisation thérapeutique de ces cellules pour les patients : le stade intermédiaire pluripotent est associé à un potentiel cancéreux et un risque d'accumulation d'aberrations génétiques lors du grand nombre de divisions cellulaires nécessaires existe $[3,4]$. Bien que les cellules iPS restent une source d'espoir pour de futures thérapies régénératives, une autre piste prometteuse se dessine : la reprogrammation directe ou transdifférenciation [15].

La transdifférenciation est la conversion directe d'un type cellulaire différencié en un autre et elle permet en principe de contourner cette étape de pluripotence tumorigène. Cependant, les étapes de ces conversions cellulaires directes restent floues et ne permettent pas d'évaluer les risques éventuels pour un patient. De plus, du fait de l'absence de traçabilité de cellules uniques in vivo, la grande majorité des modèles utilisés utilisent des cellules cultivées in vitro, ce qui ne permet pas d'aborder ce processus de transdifférenciation dans un contexte physiologique. Plusieurs questions restent en suspens : la transdifférenciation passe-t-elle par une identité intermédiaire mixte, ou bien un état intermédiaire pluripotent? Présente-t-elle les mêmes limitations que celles évoquées pour les cellules iPS? Est-elle une piste sûre pour de futures applications thérapeutiques?

\section{Caenorhabditis elegans : un modèle} puissant pour adresser ces questions De façon remarquable, des événements de transdifférenciation existent naturellement chez différents organismes, notamment chez Caenorhabditis elegans où une cellule rectale (nommée $y$ ) se transforme en motoneurone (appelé PDA) au cours du développement larvaire [5]. En effet, la cellule y fait partie du rectum, un tube composé de six cellules arrangées par paires en trois anneaux (Figure 1). Vers la fin du stade larvaire Ll, Y migre antérieurement et se transforme en motoneurone, sans faire intervenir de division cellulaire (Figure 1). Ce processus fournit un modèle in vivo idéal pour élucider les mécanismes soustendant la transdifférenciation dans un contexte physiologique. Grâce à sa 


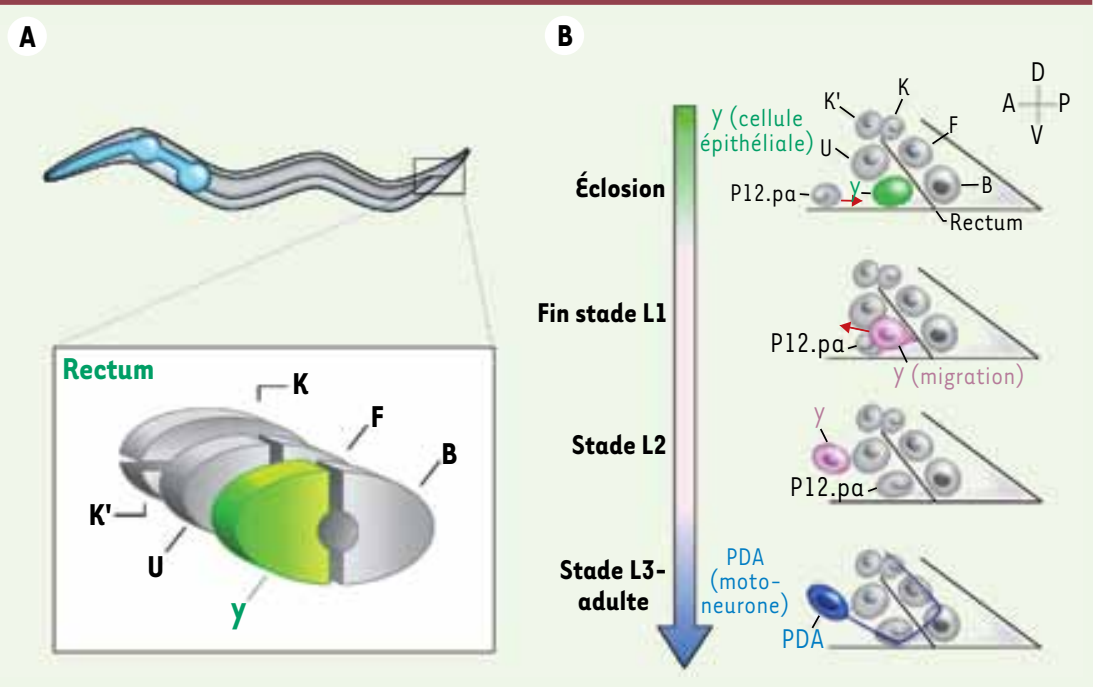

nombreuse descendance et à son temps de génération court ( 3 jours), C. elegans se prête parfaitement à des approches génétiques non biaisées pour identifier de nouveaux acteurs moléculaires. Ce ver possède un nombre fini de cellules au stade adulte: 959 cellules somatiques chez l'hermaphrodite. La transparence du nématode a permis d'établir la filiation de chaque cellule (lignage cellulaire) depuis le zygote jusqu'à l'adulte $[6,7]$, et notamment celle de la cellule y. Par conséquent, C. elegans s'impose comme un excellent modèle d'étude de reprogrammation directe in vivo à l'échelle d'une cellule.

\section{Le processus de transdifférenciation} transite par une étape dédifférenciée sans division cellulaire intermédiaire Un crible de mutagenèse aléatoire nous a permis d'identifier un certain nombre d'acteurs impliqués dans la transformation de $Y$ en PDA [8], en particulier UNC-3, un facteur de transcription conservé (Collier/Olf-1/EBF [9]) impliqué dans la différenciation neuronale. Pour analyser les étapes de la transformation cellulaire d'une cellule rectale en un neurone, notre équipe a utilisé comme modèle d'étude les mutants unc-3 [10]. Grâce à l'utilisation d'un marqueur de $y$ permettant de suivre sa migration hors du rectum, les auteurs ont montré que les premières étapes de la transdifférenciation de y (initiation et rétraction du rectum, migration) se déroulent de manière identique chez le ver sauvage (WT) et le mutant. Chez les mutants unc-3, Y n'adopte pas non plus l'identité d'une autre cellule: l'analyse de marqueurs de l'endoderme, de l'hypoderme, des muscles ou même d'autres types de neurones a exclu cette possibilité ; le processus de transdifférenciation de $Y$ en PDA est bloqué chez ces mutants à un stade intermédiaire, qui a été appelé $Y .1$.

Afin de déterminer l'identité cellulaire de l'intermédiaire $y .1$, nous avons analysé l'expression de marqueurs des celIules épithéliales et rectales ou bien de marqueurs pan-neuroblastiques et pan-neuronaux. Cette étude a montré que la cellule y.l n'a plus aucune caractéristique épithéliale ou rectale et s'est engagée vers une destinée neurale bien qu'elle n'ait pas encore acquis une morphologie spécialisée de neurone. De plus, l'étude détaillée de la dynamique d'expression de marqueurs moléculaires durant la transdifférenciation de $y$ en PDA chez les animaux sauvages montrent que la cellule $y$ perd d'abord son identité épithéliale (intermédiaire nommé y.0) avant d'acquérir des caractéristiques de cellule à destinée neurale (y.1) (Figure 2A). Ainsi, au moins deux étapes intermédiaires existent entre la cellule rectale $Y$ et le neurone PDA, et ce
Figure 1. Transdifférenciation d'une cellule du rectum nommée $y$ en un neurone appelé PDA. A. Localisation de $Y$ dans le rectum de $C$. elegans à l'éclosion. Le rectum, un organe vital, est un tube formé par 6 cellules nommées $Y$ et $B$, $U$ et $F$, et $K$ et $K$; en bleu, le pharynx, qui marque le côté antérieur du ver; en vert, la cellule $Y$, qui forme un anneau avec la cellule B. B. Migration de la cellule $Y$ hors de l'épithélium rectal lors du second stade larvaire (L2). La cellule nommée P12.pa, qui vient de naître, remplace $y$ pour reformer le tube rectal. $Y$ se transdifférencie en motoneurone PDA, représenté ici avec son axone, au troisième stade larvaire (L3). À gauche, une échelle figurant les stades du développement concernés. Antérieur est à gauche, et ventral en bas.

même en absence de division cellulaire. De plus, la première étape ressemble à une dédifférenciation : la reprogrammation directe ne passe donc pas par une identité mixte. Ceci peut représenter un mécanisme de protection de l'organisme puisque l'expression simultanée de plusieurs types cellulaires a été observée dans divers cancers [11]. Enfin, la redifférenciation par étapes en un neurone PDA semble mimer le processus de différenciation neuronale embryonnaire.

\section{Le caractère dédifférencié}

\section{de l'intermédiaire Y. 0}

n'est pas couplé à un état multipotent La reprogrammation de $y$ en PDA transite par un stade intermédiaire qui semble dédifférencié, ce qui suscite une interrogation cruciale: le changement d'identité passerait-il dans ce modèle par un stade pluripotent à I'instar de la reprogrammation produisant les cellules iPS? Pour répondre à cette question importante, nous avons testé la plasticité des états intermédiaires Y.0 et Y.l. À différentes étapes de la transition de $Y$ en PDA, nous avons forcé dans y l'expression de facteurs de transcription suffisant à l'induction de différents types cellulaires (inducteurs musculaires, intestinaux, épithéliaux, de neurones GABAergiques). Ces molécules peuvent induire la reprogrammation de blastomères dans l'embryon, 


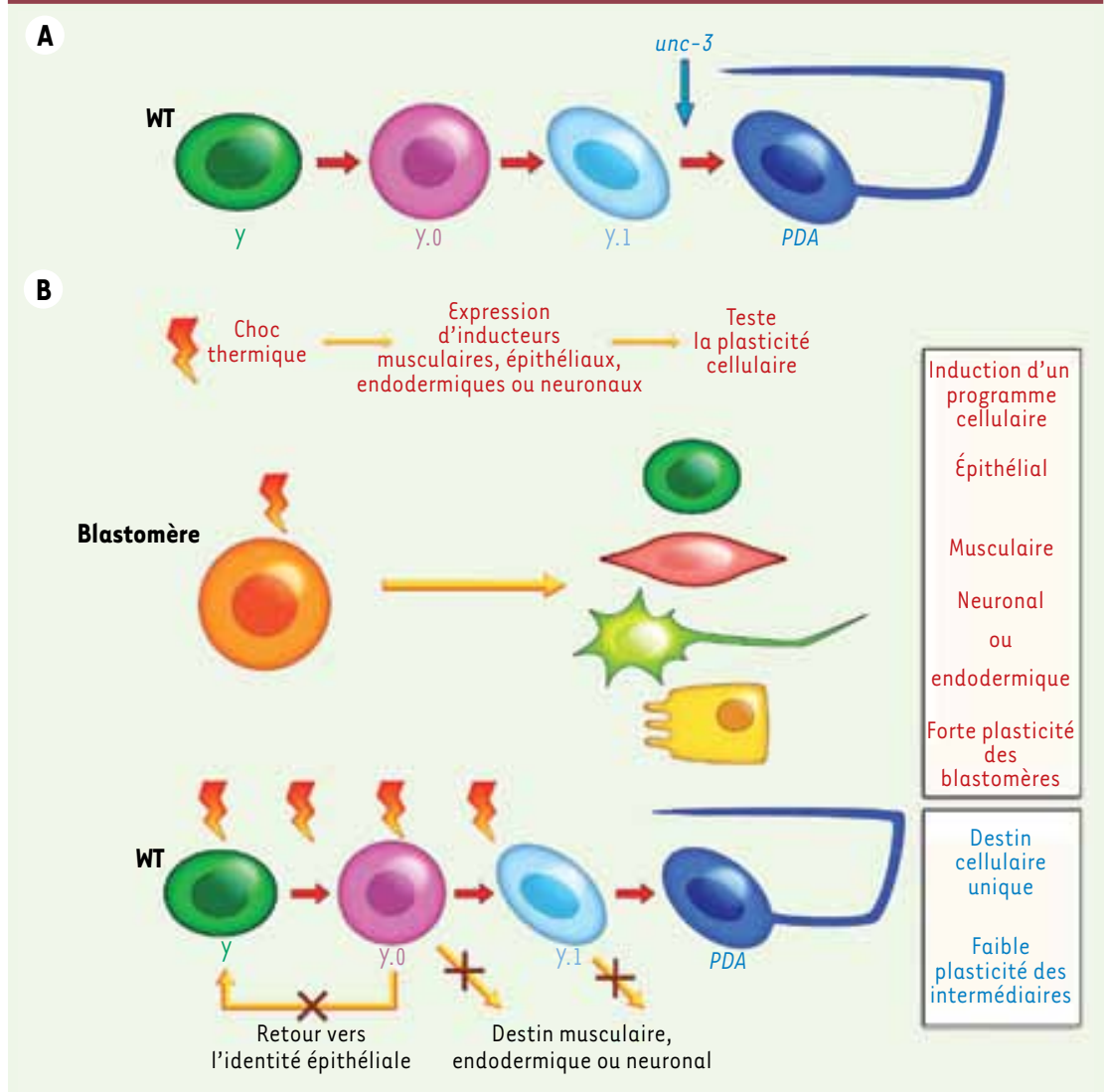

Figure 2. Découplage entre dédifférenciation et multipotence in vivo. A. Modèle de transdifférenciation de $y$ en PDA. Y transite par un stade (dédifférencié) qui a perdu toute caractéristique épithéliale et rectale, sans avoir acquis de caractéristiques neurales; ce stade est représenté ici par Y.0. La redifférenciation de Y s'effectue ensuite par étapes. La cellule s'engage d'abord vers un destin neural (intermédiaire appelé $y .1$ ) puis se transforme enfin en motoneurone d'identité PDA. Dans les mutants unc-3, la reprogrammation de $y$ est stoppée avant l'étape finale, résultant en un intermédiaire Y.l stable. B. Test de plasticité sur les intermédiaires de la transdifférenciation de $y$ en PDA. Les lignées utilisées possèdent un transgène contenant un facteur de transcription, sous contrôle d'un promoteur de choc thermique, qui est suffisant pour l'induction d'un destin cellulaire: soit $h l h-1$ (spécification musculaire), end-1 (spécification intestinale), lin-26 (spécification épithéliale), ou unc-30 (spécification en neurone

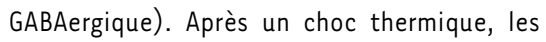
blastomères de l'embryon précoce $C$. elegans adoptent le destin cellulaire dicté par le trans-

gène, mettant en évidence leur forte plasticité. L'expression forcée de ces inducteurs dans les intermédiaires de la conversion de $Y$ en PDA ne change pas le programme initial : Y devient toujours PDA. Aucun des intermédiaires n'est donc pluripotent malgré l'étape dédifférenciée Y.0. De plus, forcer un retour vers une identité épithéliale de Y.0 ou Y.l n'est pas possible, suggérant que, dans un contexte physiologique, le potentiel cellulaire associé à chaque étape est contrôlé de façon stricte.

mais à aucun moment, chez le ver sauvage ou le mutant unc-3, l'expression de ces déterminants cellulaires n'a pu rediriger $y$ ou l'un de ses intermédiaires vers un autre destin (Figure 2B). De façon intéressante, il n'est pas non plus possible de forcer le retour en arrière de Y.o ou Y.l vers l'ancienne identité épithéliale. Ceci indique que les transitions entre les différentes identités cellulaires que prend $y$ pour devenir PDA sont strictement contrôlées in vivo. Cet aspect pourrait avoir été conservé lors d'autres évènements de plasticité cellulaire, puisqu'une étude récente a montré que les cellules qui régénèrent les membres chez l'axolotl proviennent d'une dédifférenciation des cellules avoisinantes qui ne deviennent pas pour autant multipotentes [12].
L'élucidation des mécanismes qui interviennent dans le contrôle strict du potentiel de Y sera l'enjeu des années à venir. La part de l'influence de l'environnement et celle des facteurs intrinsèques à la cellule y dans la transdifférenciation devront être précisées. Une étude récente chez le rat révèle le rôle essentiel du contexte physiologique dans le contrôle de l'identité cel- $\quad(\rightarrow)$ Voir Brève, lulaire ou sa reprogram- page 258 de ce mation $[13,18](\rightarrow)$. numéro D'autre part, l'extinction puis la mise en place de deux programmes d'expression cellulaires distincts pourraient impliquer une restructuration de la chromatine. À l'appui de cette hypothèse, la plupart des mutants que nous avons identifiés soulignent le rôle important de complexes nucléaires contenant des facteurs connus pour maintenir la pluripotence des cellules ES, et d'activités épigénétiques. Enfin, la transdifférenciation apparaît d'ores et déjà comme une voie d'avenir pour la médecine régénérative, puisqu'elle ne nécessite pas de division cellulaire et ne produit pas d'intermédiaire multipotent potentiellement tumorigène. $\diamond$

Direct reprogramming: a little worm unveils the cellular transitions

\section{REMERCIEMENTS}

Les auteurs remercient Elisabeth GeorgesLabouesse pour sa lecture critique du manuscrit. Le travail décrit a été soutenu par des financements de l'AFM, la FRM, ATIP-CNRS et l'ARC.

\section{CONFLIT D’INTÉRÊTS}

Les auteurs déclarent n'avoir aucun conflit d'intérêts concernant les données publiées dans cet article. 


\section{RÉFÉRENCES}

1. Gurdon JB, Elsdale TR, Fischberg M. Sexually mature individuals of Xenopus laevis from the transplantation of single somatic nuclei. Nature $1958 ; 182: 64-5$.

2. Takahashi K, Yamanaka S. Induction of pluripotent stem cells from mouse embryonic and adult fibroblast cultures by defined factors. Cell $2006 ; 126: 663-76$.

3. Gurdon JB, Melton DA. Nuclear reprogramming in cells. Science 2008 ; 322 : 1811-5.

4. Mayshar $\mathrm{Y}$, Ben-David U, Lavon N, et al. Identification and classification of chromosomal aberrations in human induced pluripotent stem cells. Cell Stem Cell $2010 ; 7: 521-31$.

5. Jarriault S, Schwab Y, Greenwald I. A Caenorhabditis elegans model for epithelial-neuronal transdifferentiation. Proc Natl Acad Sci USA 2008 ; 105 : 3790-5.

6. Sulston JE, Horvitz HR. Post-embryonic cell lineages of the nematode, Caenorhabditis elegans. Dev Biol 1977 ; $56: 110-56$

7. Sulston JE, Schierenberg $\varepsilon$, White JG, Thomson $J N$. The embryonic cell lineage of the nematode Caenorhabditis elegans. Dev Biol 1983; $100: 64-119$.
8. Zuryn S, Le Gras S, Jamet K, Jarriault S. A strategy for direct mapping and identification of mutations by whole-genome sequencing. Genetics 2010 ; $186: 427-30$.

9. Dubois L, Vincent A. The COE--Collier/Olfl/EBF--transcription factors: structural conservation and diversity of developmental functions. Mech Dev 2001 $108: 3-12$.

10. Richard J, Zuryn S, Fischer N, et al. Direct in vivo cellular reprogramming involves transition through discrete, non-pluripotent steps. Development 9 mars 2011 (online).

11. Zhang $\mathrm{Q}$, Fan $\mathrm{H}$, Shen J, et al. Human breast cancer cell lines co-express neuronal, epithelial, and melanocytic differentiation markers in vitro and in vivo. PLoS One $2010 ; 5$ : e9712.

12. Kragl M, Knapp D, Nacu E, et al. Cells keep a memory of their tissue origin during axolotl limb regeneration. Nature $2009 ; 460: 60-5$

13. Bonfanti P, Claudinot S, Amici AW, et al. Microenvironmental reprogramming of thymic epithelial cells to skin multipotent stem cells. Nature 2010 ; $466: 978-82$
14. Coulombel L. Reprogrammation nucléaire d'une cellule différenciée : on efface tout et on recommence. Med Sci (Paris) $2007 ; 23: 667-70$

15. De Vos J. Transdifférenciation induite : la plasticité cellulaire revisitée. Med Sci (Paris) 2010 ; 26 : 904-6.

16. Zipori D. À la recherche d'une définition moléculaire plus que descriptive pour les cellules souches. Med Sci (Paris) $2011 ; 27: 303-7$

17. Charbord P, Casteilla L. La biologie des cellules souches mésenchymateuses d'origine humaine. Med Sci (Paris) $2011 ; 27: 261-8$

18. Coulombel L. Peau et thymus: une cellule souche épithéliale commune à la défense du soi. Med Sci (Paris) $2011 ; 27: 258$

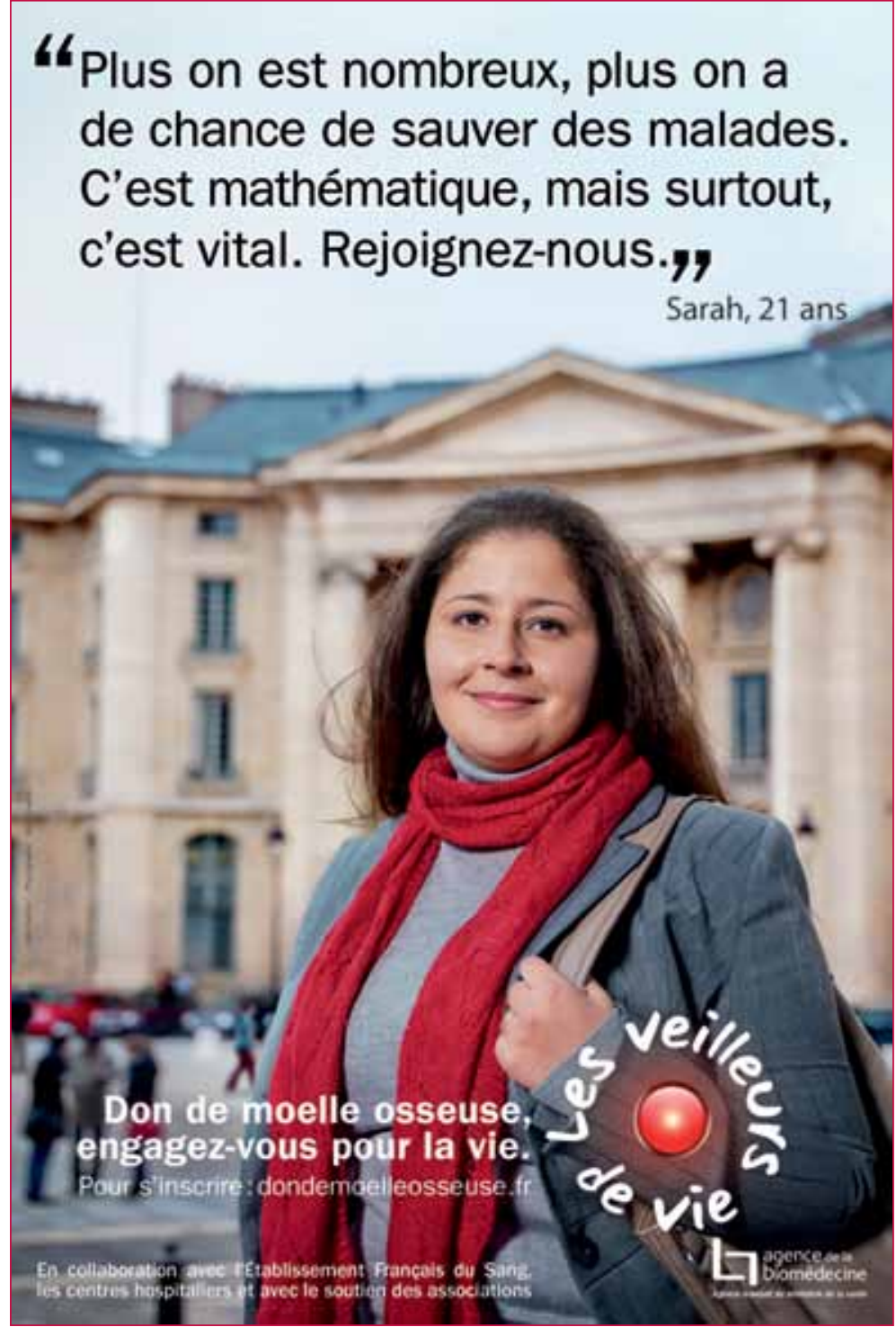

TIRÉS À PART

M.C. Morin et S. Jarriault 